\title{
Vacuum Spacetimes with a spacelike Killing vector
}

\section{Duong Phan}

The principal aim of this thesis is to find exact solutions of vacuum gravitational fields with a spacelike Killing vector.

It is well known that the formulation of the vacuum Einstein equations for the case where there is a non-null Killing vector may be reduced to a three dimensional problem in the quotient space generated by the trajectories of the Killing vector [2].

Because there is a quotient 3 -space, the triad formalisms $[3,10]$ are used and the vacuum field equations for the following three cases are obtained:

(1) the twistfree case,

(2) the null Papapetrou case, and

(3) the general case with twist.

The methods of symmetry groups $[6,8,9]$ are then to be used for group-invariant solutions. All solutions obtained are classified [7] according to Petrov types and group of motions. Because of the length and the complexity of closed-form solutions, it would not be possible to carry out the analysis by hand calculations. We use symbol manipulation (computer algebra) programs instead, and REDUCE [4] is constantly used.

In case (1) we first prove that there exists a plane of Ricci collineations tangent to the equipotential surfaces. This fact is then used to make a suitable choice of basis triad in the quotient 3-space, depending on whether the equipotential surfaces are timelike, spacelike or null. By this means the vacuum field equations for these three separate cases are able to be written in concise forms. In the case of null equipotential surfaces, the general solution of the field equations is found. This solution is of Petrov type III of Kundt's class. In the two other cases, certain assumptions about the solutions are shown to lead to Kasner type solutions and a cylindrically and reflectionally symmetric solution.

The invariance group of the fundamental field equations is determined in the case where the equipotential surfaces are spacelike, thus enabling a deeper analysis of the structure of the solutions to be carried out. The invariance group is shown to have a

Received 30th September, 1992

Thesis submitted to the University of Sydney, January 1892. Degree approved, September 1992. Supervisor: Associate Professor E.D. Fackerell.

Copyright Clearance Centre, Inc. Serial-fee code: 0004-9729/93 \$A2.00+0.00. 
two-parameter subgroup and two distinct infinite groups. These can be used to find group invariant solutions and the method of group reduction is used to make further progress. By this means group invariant solutions which admit two commuting Killing vectors are discussed.

In the case where two successive reductions using both a finite subgroup and an infinite subgroup are carried out, a non-Painlevé type ordinary differential equation of second order [5] is obtained. The solutions determined by this ordinary differential equation are of Petrov type $I$ and admit, in general, two non-commuting Killing vectors. In this case it is possible, in principle, to derive a series solution to any given order, though there is no simple recurrence relation. Actual calculations are carried out to the fifth order.

Case (2), the null Papapetrou case, is shown to be able to be reduced to case (1) by the introduction of a suitable new potential. As an example, one of the Kasner solutions and the cylindrically and reflectionally symmetric solution obtained for the twistfree case are transformed to a Papapetrou solution which admits a Bianchi $G_{3} I I$ group of motions and a cylindrically symmetric solution, respectively. These Papapetrou solutions are in fact the Ehlers' transforms of the twistfree solutions.

In case (3), the case with twist, the quotient 3-space admits a Ricci collineation. Suitable basis triad in the quotient 3-space is then can be built, depending on whether this collineation is null or not. The case where the collineation is null has been discussed by Fackerell and Kerr [1]. For a non-null Ricci collineation, vacuum gravitational fields are obtained for the case where this direction is timelike. (Vacuum field equations for this direction to be spacelike can be built in a similar way).

The analysis of the symmetry group of the field equations shows that there are three finite and two infinite isovector fields. As in the twistfree case, solutions with a $G_{2} I$ and $G_{2} I I$ are discussed. A system of four ordinary differential equations of first order and first degree is obtained as a result of two successive reductions using a combination of a finite and an infinite isovector fields. Solutions determined by these ordinary differential equations are also Petrov type $I$ and in general, admit a homothetic motion and two non-commuting Killing vectors.

\section{REFERENCES}

[1] E.D. Fackerell and R. Kerr, 'Einstein vacuum field equations with a single non-null Killing vector', Gen. Relativity Gravitation 3 (1991), 861-876.

[2] R. Geroch, 'A method for generating solutions of Einstein's equations', J. Math. Phys. 12 (1971), 918-924.

[3] G.S. Hall, T. Morgan and Z. Perjés, 'Three-dimensional spacetimes', Ger. Relativity Gravitation 19 (1987), 1137-1147. 
[4] A.C. Hearn, REDUCE User's manual version 3.3 (The RAND Corporation, Santa Monica, 1988).

[5] E.L. Ince, Ordinary differential equations (Dover Publications Inc., 1956).

[6] P.H.M. Kersten, Infinitesimal symmetries: a computational approach, Ph.D. thesis (University of Twente, Enschede, The Netherlands, 1985).

[7] D. Kramer, H. Stephani, E. Herlt and M. MacCallum, Exact solutions of Einstein's field equations (Cambridge University Press, 1980).

[8] P.W. Olver, Applications of Lie groups to differential equations (Springer-Verlag, Berlin, Heidelberg, New York, 1986).

[9] L.V. Ovsiannikov, Group analysis of differential equations (Academic Press, 1982).

[10] Z. Perjés, 'SU(1,1) spin coefficients', Acta Phys. Acad. Scientiarum Hungaricae 32 (1972), 207-220.

School of Mathematics and Statistics

The University of Sydney

New South Wales 2006

Australia 